\title{
Nouvelles du Comité central
}

Bureau d'expertises extrajudiciaires - En 2013, le nombre de cas traités par le Bureau d'expertises extrajudiciaires a connu une légère hausse. Cela comprend cependant en partie des cas encore en suspens datant de 2012.

Accord FMH-Swisscom - Dans le cadre des négociations avec Swisscom au sujet de la HPC, le Comité central (CC) a validé le règlement transactionnel relatif au contrat d'entreprise, de même que le contrat d'exploitation qui a été négocié.

Initiative parlementaire - L'initiative parlementaire «LAMal. Accorder plus d'autonomie au personnel soignant» prévoit notamment d'élargir les compétences du personnel soignant. Si les associations professionnelles consultées soutiennent un élargissement de la collaboration interprofessionnelle dans le domaine de la santé, la plupart d'entre elles ne sont en revanche pas favorables à l'initiative. Elles demandent en particulier une délimitation claire entre les prestations fournies de manière autonome par le corps médical et celles fournies par les soignants.

Conférence suisse de santé publique - La Conférence suisse de santé publique qui se tiendra cette année à Olten sera consacrée au thème «Public Mental Health». La FMH organisera à cette occasion un atelier sur la prévention au cabinet médical qui por- tera sur des thèmes d'actualité. Le CC accepte de soutenir financièrement la conférence.

Révision du droit de la prescription - Le Conseil fédéral demande une amélioration du droit de la prescription et notamment l'instauration d'un délai de prescription absolu de trente ans en cas de dommage corporel. Or, pour les traitements médicaux ordinaires, un délai de 10 ans suffit. Le CC propose donc de réserver le délai de 30 ans aux seuls dommages différés, soit aux dommages consécutifs à une exposition à l'amiante ou aux rayonnements. Par ailleurs, le Parlement doit clarifier si la protection en matière d'assurance protection civile est garantie et si la conservation des dossiers médicaux est possible pour une durée de trente ans.

Rubrique «Service d'urgence» - Les inscriptions dans la catégorie «Médecins» de l'annuaire téléphonique sont régies par une convention avec local.ch. Or selon ce fournisseur, des problèmes de délimitation sont apparus dans la rubrique en ligne «Service d'urgence», entre les inscriptions de la catégorie «Médecins» et les inscriptions en dehors de cette dernière. local.ch demande donc à la FMH de compléter la convention en ajoutant par ex. le terme «médecin» ou «médical» à la rubrique «Service d'urgence». Le CC accepte de nommer la rubrique «Service d'urgence Médecins de garde».

\section{ENVOYEZ VOS PROJETS AVANT LE 30 AVRIL 2014}

Le Swiss Quality Award récompense les meilleures innovations en matière de qualité dans le système de santé. Participez et enregistrez votre projet sur le site www.swissqualityaward.ch. 